\title{
Preparation of fusion materials based on ionic liquids and cationic gold nanoparticles
}

\author{
Takuya Nakashima, Yu Hayakawa, Midori Mori and Tsuyoshi Kawai
}

Fusion materials based on cationic gold nanoparticles mixed with ionic liquids were prepared. An anion exchange of the surfacecapping ligand on the gold nanoparticles from a halogen anion to bis(trifluoromethanesulfonyl)amide $\left(\mathrm{Tf}_{2} \mathrm{~N}\right)$ in an aqueous solution afforded nanoparticles showing infinite miscibility with $\mathrm{Tf}_{2} \mathrm{~N}$-based ionic liquids. The thermal decomposition temperature of the gold nanoparticle was elevated by $100^{\circ} \mathrm{C}$ after the anion-exchange process. The ionic liquid-like structure of the surfacecapping ligand with $\mathrm{Tf}_{2} \mathrm{~N}$ anions led to a glassy solid material with a densely packed assembly of nanoparticles, in which a portion of nanoparticles formed superlattices. A fusion material of ionic liquid and gold nanoparticles with a gold content as high as $\mathbf{4 0} \mathrm{wt} \%$ was obtained by the co-solvent evaporation method using acetone. The stable dispersion of gold nanoparticles in the fusion materials with a high gold content was confirmed by the clear appearance of the plasmon absorption of gold nanoparticles in an optical microscopic image, as well as in an absorption spectrum. The use of an ionic liquid-based monomer yielded a gold nanoparticle-ionic liquid polymer composite, in which the gold nanoparticles showed a high thermal stability.

Polymer Journal (2015) 47, 171-176; doi:10.1038/pj.2014.103; published online 19 November 2014

\section{INTRODUCTION}

Room-temperature ionic liquids (ILs) have been attracting much interest as environmentally benign solvents for organic chemical reactions, separations, and electrochemical applications. ${ }^{1-5}$ Recent interest has focused on their use in inorganic synthesis, ${ }^{6-8}$ hybridization with nanomaterials ${ }^{9-11}$ and molecular self-assembly. ${ }^{12-17}$ The inherent self-assembling properties of ILs, derived from interionic hydrogen bonding interactions ${ }^{18,19}$ and their amphiphilic nature, ${ }^{20,21}$ most likely have an important role in the solubilization of nanomaterials, including molecular self-assemblies, as well as in the synthesis of nanomaterials with fine structures. ILs therefore enable the direct in situ synthesis of metal nanoparticles (NPs) without the addition of extra stabilizers through the chemical reduction of metal ions ${ }^{22,23}$ and sputter depositions. ${ }^{24}$ The reorganization of the hydrogen bond network and the generation of nanostructures with ionic components were presumed to form a protecting layer on the surface of NPs, providing both steric and electrostatic protection against agglomeration. $^{23}$ In addition, the fusion of the self-assembling property of ILs and functional inorganic nanomaterials is expected to lead to a futuristic material, in which both components synergistically interact to construct a hierarchically assembled structure. Furthermore, the unique characteristics of ILs, such as high ionic conductivity, thermal stability and extremely low vapor pressure, which could be tuned by changing the anion identity and cation substituents, make ILs an excellent partner to build composite materials with nanomaterials. ${ }^{25-28}$ For example, IL-silica NP hybrids have been demonstrated as an electrolyte for batteries. ${ }^{29}$
A wide range of metal and semiconductor NPs, with or without surface-capping ligands, have been reported to be well dispersed in ILs. Because electrostatic charge stabilization, which usually accounts for the colloidal stability of NPs in water, is considered to be insufficient owing to the high ionic strength in ILs; IL-based solvation might be responsible for colloidal stability in ILs. ${ }^{29}$ However, the dynamically fluctuating organized structure of ILs ${ }^{20}$ and ill-defined interactions ${ }^{30,31}$ between the surface of NPs and IL components may hamper the stable dispersion of bare NPs in ILs with a high NP content, especially at high temperatures. In this context, NPs with imidazoliumfunctionalized surface-capping ligands ${ }^{32-34}$ or polymers ${ }^{35}$ showed better stability in ILs. Some NPs stabilized by IL-based ligands showed liquid-like behavior at room temperature in the absence of solvent media. ${ }^{36-38}$ Conventionally, such NPs have been prepared in a media, such as water or an organic solvent, and then transferred into an IL via the modification of the ligands. Recently, we reported the spontaneous phase transfer of CdTe NPs capped with simple cationic ligands, 2-(dimethylamino)ethanethiol hydrochloride (DMAET) or thiocholine bromide, from aqueous solutions to ILs. ${ }^{39-41}$ The cationic CdTe NPs showed remarkable stability and improved photoluminescent properties after the phase transfer in the ILs. The use of these cationic ligands afforded NP-IL-based polymer composites at volume fractions of NPs as high as $1 \mathrm{vol} \%$ (3.6 wt $\%$ ), which demonstrated high-order optical nonlinearlities. ${ }^{42,43}$

In the present paper, we report the preparation of hybrid materials based on ILs mixed with cationic gold NPs. To achieve a very-high loading content of NPs in ILs, cationic NPs with bis(trifluoromethanesulfonyl)amide $\left(\mathrm{Tf}_{2} \mathrm{~N}\right)$ anions were prepared. The cationic gold NPs 
with $\mathrm{Tf}_{2} \mathrm{~N}$ anions formed a glassy film when cast from an acetone solution and resulted in composite materials with ILs that have a gold content as high as $40 \mathrm{wt} \%$. The gold NPs exhibited a high thermal stability in an IL-based polymer.

\section{EXPERIMENTAL PROCEDURE}

\section{Materials and methods}

1-Butyl-3-methylimidazolium $\mathrm{Tf}_{2} \mathrm{~N}\left(\right.$ bmimTf $\left._{2} \mathrm{~N}\right)$ and lithium $\mathrm{Tf}_{2} \mathrm{~N}$ were purchased from Kanto Chemical Co., Inc. Tokyo, Japan. 2-(Methacryloyloxy) ethyltrimethylammonium $\mathrm{Tf}_{2} \mathrm{~N}$ (MOE-200) was purchased from Piotrek Co., Ltd. Shiga, Japan. Hydrogen tetrachloroaurate(III) tetrahydrate was supplied by Wako Pure Chemical Industries Ltd. Osaka, Japan. DMAET and Irgacure 184 (1-hydroxycyclohexyl phenyl ketone) were obtained from TCI Co. Ltd., Tokyo, Japan. These chemicals were used as received. Diethylene glycol dimethacrylate was obtained from TCI Co. Ltd and used after a simple distillation process. Absorption spectra in solution were studied with a JASCO V-670 spectrophotometer (JASCO, Tokyo, Japan). Thermogravimetric analysis was carried out using a TG-DTA6200 (SII Technology, Chiba, Japan). Transmission electron microscopy (TEM) observation was performed with a JEM-2200FS (JEOL, Tokyo, Japan). Scanning electron micrographs were obtained by using an SU9000 (Hitachi, Tokyo, Japan). Small angle X-ray scattering profiles were recorded using an RINT-TTR III/NM X-ray diffractometer (Rigaku, Tokyo, Japan) in transmission mode. Absorption spectra of concentrated fusion materials were measured using a BX-51 (Olympus, Tokyo, Japan) polarizing microscope connected to a Hamamatsu PMA-11 (Hamamatsu Photonics, Shizuoka, Japan) photodetector with an optical fiber.

\section{Synthesis of DMAET-capped gold NPs and fusion materials}

Fusion materials based on gold NPs and ILs were prepared according to Scheme 1. The gold NPs were synthesized by the chemical reduction of aqueous $\mathrm{Au}^{3+}$ in the presence of the cationic thiol DMAET. To a solution of hydrogen tetrachloroaurate(III) tetrahydrate $(0.33 \mathrm{~g}, 0.80 \mathrm{mmol})$ and DMAET $(0.28 \mathrm{~g}$, $2.4 \mathrm{mmol})$ in deionized water $(67 \mathrm{ml})$, an aqueous solution $(6.7 \mathrm{ml})$ of sodium borohydride $\left(\mathrm{NaBH}_{4}\right)(20 \mathrm{mg}, 0.53 \mathrm{mmol})$ was added under vigorous stirring in an ice bath. After stirring for $1 \mathrm{~h}$, the insoluble sediment was removed through centrifugation, followed by decantation. DMAET-capped gold NPs in the supernatant were purified via reprecipitation by the addition of acetone $(300 \mathrm{ml})$. The precipitate was freeze-dried to give $82 \mathrm{mg}$ of the DMAET-capped gold NPs in a $44 \%$ yield (in terms of the conversion of $\mathrm{Au}^{3+}$ ). $50 \mathrm{mg}$ of the DMAET-capped gold NPs were redispersed in deionized water $(10 \mathrm{ml})$, to which an aqueous solution of lithium $\mathrm{Tf}_{2} \mathrm{~N}(70 \mathrm{mg}, 0.24 \mathrm{mmol}$ ) was added to exchange the counter anion of DMAET from $\mathrm{Cl}^{-}$to $\mathrm{Tf}_{2} \mathrm{~N}^{-}$. DMAET- $\mathrm{Tf}_{2} \mathrm{~N}$ passivated gold NPs were then obtained as a precipitate. The precipitated gold NPs were freeze-dried by a lyophilizer to give $46 \mathrm{mg}$ of cottony powder in a $61 \%$ yield.

The DMAET-Tf ${ }_{2} \mathrm{~N}$ passivated gold NPs were dispersed in acetone with a certain amount of IL (bmimTf ${ }_{2} \mathrm{~N}$ or MOE-200, Scheme 1). The fusion materials were obtained after the evaporation of acetone in vacuo. The fusion material with MOE-200 was further polymerized by photoradical polymerization. To a mixture of gold NPs and MOE-200, a diethylene glycol dimethacrylate (20 wt $\%$ ) cross-linker and an acetone solution of Irgacure 184 (1 wt $\%)$ were added, the acetone was evaporated in vacuo and then the mixture was degassed. The monomer cocktail was cast on a glass substrate loaded with a silicone film spacer (thickness: $100 \mu \mathrm{m}$ ) and was covered with another glass substrate. The photopolymerizable nanocomposite film was exposed to UV light from a mercury-xenon lamp $(200 \mathrm{~W})$ through a UV-transmitting filter at room temperature to prepare a uniformly cured polymer nanocomposite film.

\section{RESULTS AND DISCUSSION}

\section{DMAET-Tf ${ }_{2} \mathrm{~N}$-capped gold NPs}

Figure 1 shows a TEM image of DMAET-protected gold NPs prepared from an aqueous solution before anion exchange. Relatively monodisperse, quasi-spherical NPs with an average diameter of $2.9 \pm 0.4 \mathrm{~nm}$ were observed. In the absorption spectrum, an apparent plasmon absorption peak appeared at $517 \mathrm{~nm}$ (Figure 2). The anion exchange of the gold NPs from chloride to $\mathrm{Tf}_{2} \mathrm{~N}$ made the NPs insoluble in water but soluble in highly polar organic solvents, such as acetone. The hydrophilic chloride anion is thought to bind tightly to the ammonium cation of DMAET and forms an aggregate in acetone, which was demonstrated by the precipitation purification procedure of DMAETcapped gold NPs using acetone. The anion exchange to $\mathrm{Tf}_{2} \mathrm{~N}$ with a higher ionic radius decreased the hydrophilicity of the counter anion, which was expected to reduce the interionic interaction and allowed ions to partially dissociate in acetone. ${ }^{44}$ The DMAET- $\mathrm{Tf}_{2} \mathrm{~N}$ passivated gold NPs also exhibited a clear plasmon absorption peak in acetone,

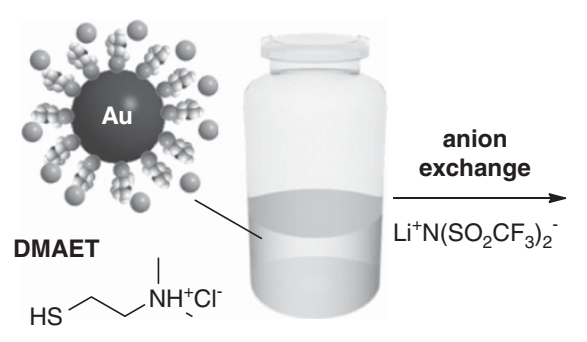

Au NP@DAMET aq.

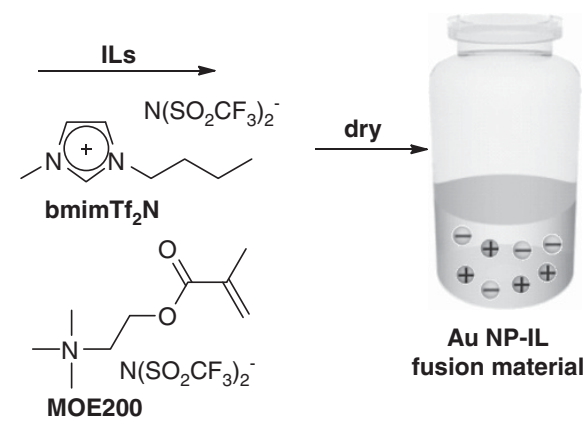

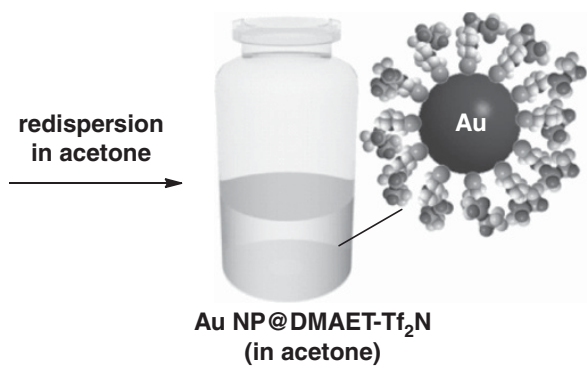

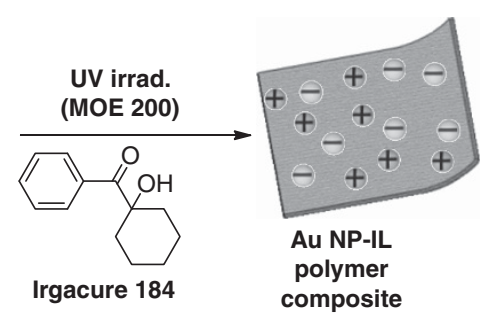

acure 18

Scheme 1 Preparation of fusion materials based on gold nanoparticles and ionic liquids. A full color version of this scheme is available at Polymer Journal online. 


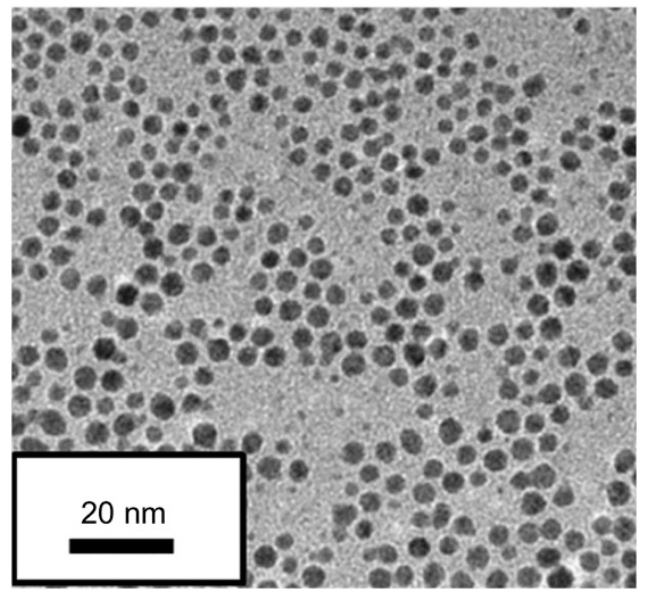

Figure 1 Transmission electron microscopy image of 2-(dimethylamino) ethanethiol hydrochloride-capped gold nanoparticles cast from an aqueous solution.

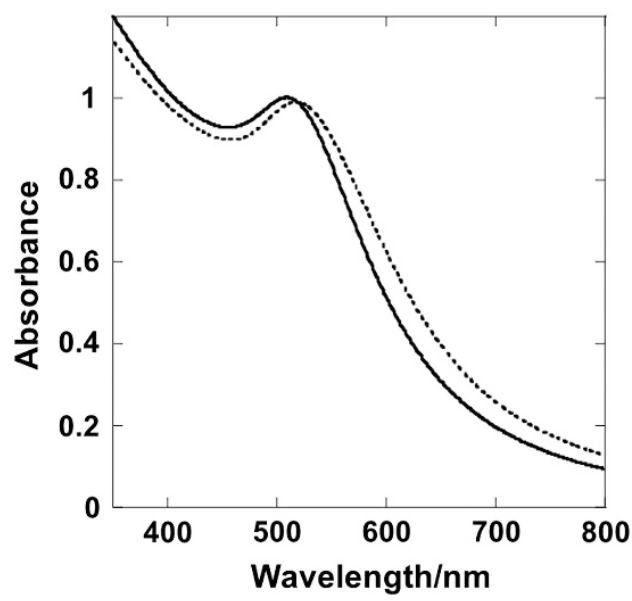

Figure 2 Absorption spectra of 2-(dimethylamino)ethanethiol hydrochloridecapped gold nanoparticles in water (dotted line) and in acetone (solid line) after anion exchange with bis(trifluoromethanesulfonyl)amide.

suggesting the stable dispersion of NPs. The peak appeared at $510 \mathrm{~nm}$, which was slightly shorter than that observed in water most likely because of the change in the permittivity of solvents. ${ }^{45}$

The acetone solution of gold NPs was cast onto a TEM grid, which was observed by TEM and scanning electron micrograph. As shown in Figure 3a, the gold NPs formed a densely packed, glassy-assembled film on the TEM grid. The separation between NPs was apparently shorter than that observed from the TEM image in Figure 1. Because the thickness of an electrical double layer is dependent on the permittivity of solvents, ${ }^{46}$ the reduced electrostatic repulsion in the acetone was responsible for the densely packed assembly. Larger particles with sizes of a few hundred nanometers were also observed in the glassy amorphous film, as shown in Figure $3 \mathrm{~b}$ (scanning electron micrograph). Interestingly, the large particles were recognized as secondary particles composed of the individual cationic gold NPs assembling into superlattice structures, which was confirmed by a high-resolution scanning electron micrograph image (Figure 3c). These supercrystalline structures could be formed during evaporation because the acetone solution for electron microscopy measurements
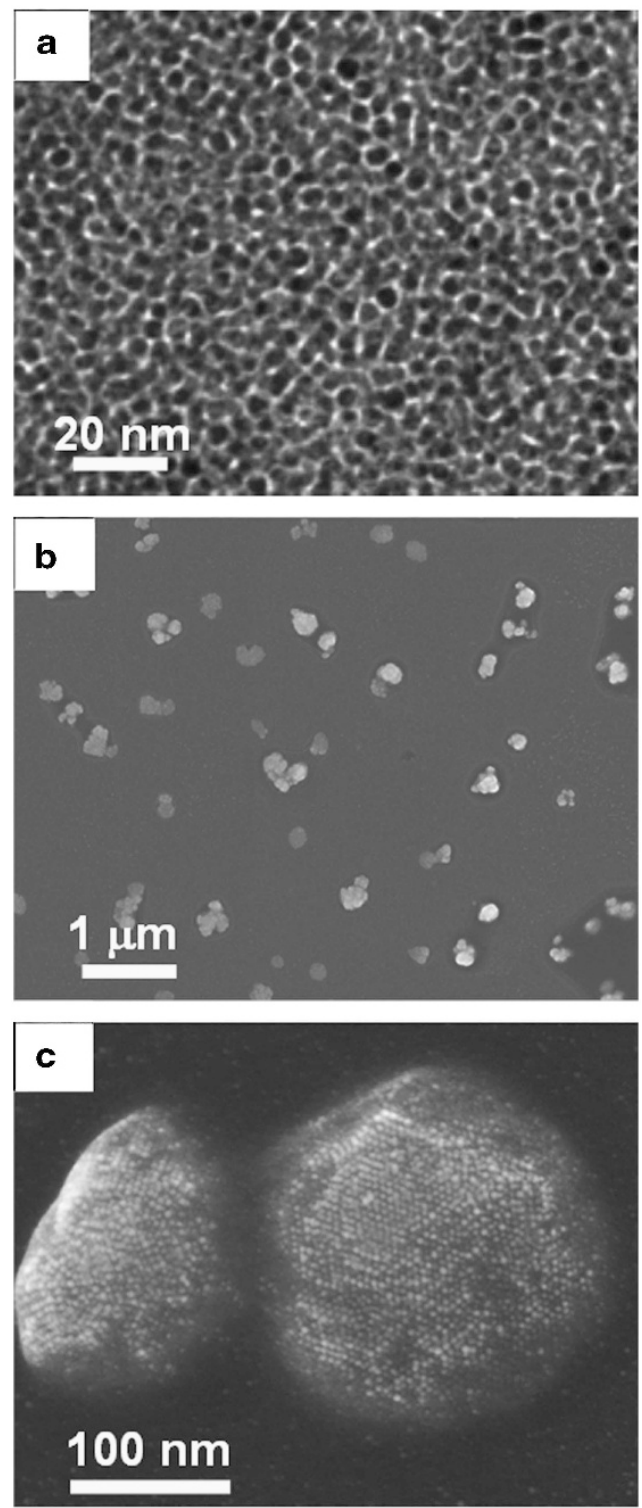

Figure 3 Electron micrographs of 2-(dimethylamino)ethanethiol hydrochloridebis(trifluoromethanesulfonyl)amide-capped gold nanoparticles prepared from an acetone solution: a transmission electron microscopy (a), scanning electron micrographs $(\mathbf{b}, \mathbf{c})$ images.

was subjected to centrifugation at $10^{4}$ r.p.m., followed by filtration with a membrane filter prior to drop casting. The elevation of NP concentration and ionic strength during the evaporation of the solvent may result in the close contact between NPs by minimizing the electrical double layer, so that the van der Waals force between NPs becomes effective. ${ }^{46}$ Furthermore, the cohesive interactions between IL-like DMAET-Tf $2 \mathrm{~N}$ surface ligands, which could give rise to the self-assembling property of ILs, should play a crucial role in the self-assembly of NPs.

The properties of DMAET- $\mathrm{Tf}_{2} \mathrm{~N}$-capped gold NPs in bulk were also investigated by thermal analytical methods. The DMAET-capped NPs before the anion-exchange process decomposed at $\sim 200^{\circ} \mathrm{C}$, which is most likely because of the high chemical reactivity of the chloride anion (Figure 4). The anion exchange to $\mathrm{Tf}_{2} \mathrm{~N}$ increased the decomposition temperature of the ligands. The mass decrease of DMAET-Tf $2 \mathrm{~N}$-capped gold NPs started from $\sim 300^{\circ} \mathrm{C}$, which was 


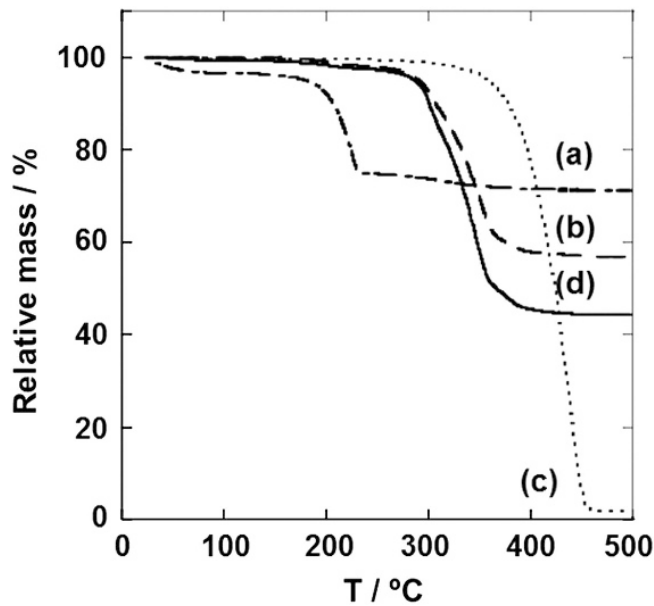

Figure 4 Thermogravimetric curves of (a) 2-(dimethylamino)ethanethiol hydrochloride-capped gold nanoparticle, (b) 2-(dimethylamino)ethanethiol hydrochloride-bis(trifluoromethanesulfonyl)amide-capped gold NP, (c) 1butyl-3-methylimidazolium bis(trifluoromethanesulfonyl)amide and (d) gold nanoparticle-1-butyl-3-methylimidazolium bis(trifluoromethanesulfonyl)amide composite.

$100^{\circ} \mathrm{C}$ higher than that of the gold NPs before anion exchange, directly indicating the higher chemical stability of $\mathrm{Tf}_{2} \mathrm{~N}^{-}$in comparison with chloride ion. The DMAET-capped gold NPs contained an organic content of $29 \mathrm{wt} \%$, corresponding to 430 ligand molecules per single NP (6.1 $\AA^{2}$ of occupied surface area per a ligand molecule). This number seems too large considering that the typical occupied surface area of a similar ligand molecule thiocholine bromide was estimated to be $22.9 \AA, 2,47$ indicating the presence of unbound excess ligand molecules. Similarly, the DMAET-Tf $2 \mathrm{~N}$-capped gold NPs possessed a $42 \mathrm{wt} \%$ portion of organic ligands, which corresponds to 280 ligand molecules per single NP and still had unbound excess ligand molecules.

Figure 5 shows differential scanning calorimetry (DSC) thermograms of DMAET-Tf ${ }_{2} \mathrm{~N}$ and gold NPs. DMAET-Tf ${ }_{2} \mathrm{~N}$ became an IL after the anion-exchange process. The free DMAET- $\mathrm{Tf}_{2} \mathrm{~N}$ showed an exothermic peak at $-47^{\circ} \mathrm{C}$ corresponding to the cold-crystallization and endothermic peaks at -7 and $7^{\circ} \mathrm{C}$, assigned as melting points, which are typical for $\mathrm{Tf}_{2} \mathrm{~N}$-based ILs. ${ }^{48}$ By contrast, the DMAET$\mathrm{Tf}_{2} \mathrm{~N}$-capped gold NPs showed no apparent DSC peak, indicating that the free excess DMAET- $\mathrm{Tf}_{2} \mathrm{~N}$ ligands had a negligible effect on the thermal behavior of the NPs. The DSC profile of the gold NPs exhibited a slight endothermic shift of the baseline at $58^{\circ} \mathrm{C}$, corresponding to the glass transition temperature, which also supported the glassy amorphous assembly of gold NPs in the solid state. There is no endothermic peak observed for the melting of the superlattice assemblies in the temperature range investigated, indicating the supercrystals were only the minor components in the solid film. The small angle X-ray scattering profile measured for the DMAET-Tf $2 \mathrm{~N}$-capped gold NPs suggested an amorphous-like assembly in the cast film, which provided a broad peak corresponding to the interparticle correlation at a distance of $4.8 \mathrm{~nm}$ and gave a little indication of a highly ordered aggregation with periodic peaks (Supplementary Figure S1).

Fusion materials of DMAET-Tf $\mathrm{f}_{2} \mathrm{~N}$-capped gold NPs and ILs The DMAET-Tf $f_{2} \mathrm{~N}$-capped gold NPs were mixed with bmim $\mathrm{Tf}_{2} \mathrm{~N}$ by a co-solvent evaporation method using acetone, because the direct

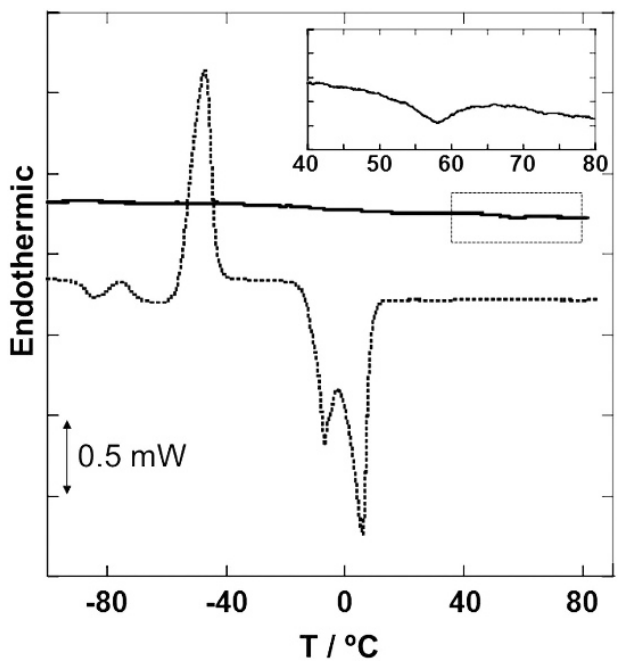

Figure 5 DSC thermograms of 2-(dimethylamino)ethanethiol hydrochloridebis(trifluoromethanesulfonyl)amide (dotted line) and 2-(dimethylamino) ethanethiol hydrochloride-bis(trifluoromethanesulfonyl)amide-capped gold nanoparticles (solid line). Inset: enlarged profile of 2-(dimethylamino) ethanethiol hydrochloride-bis(trifluoromethanesulfonyl)amide-caped gold nanoparticles.

dispersion of NPs resulted in the partial solubilization of NPs in ILs because of the high viscosity of ILs. BmimTf ${ }_{2} \mathrm{~N}$ was thermally stable up to $350{ }^{\circ} \mathrm{C}$ (Figure $4 \mathrm{c}$ ), whereas the concentrated composite with a $40 \mathrm{wt} \%$ gold content showed decomposition above $300^{\circ} \mathrm{C}$ (Figure $4 \mathrm{~d}$ ). The decomposition temperature of the composite was identical with that of DMAET-Tf ${ }_{2} \mathrm{~N}$-capped gold NPs without IL (Figure $4 \mathrm{~b}$ ), which indicated that the decomposition started from the NPs in the composite.

The gold NPs at a given composition were homogeneously dispersed in bmimTf $_{2} \mathrm{~N}$ after the evaporation of acetone. Figure $6 \mathrm{a}$ shows the visual appearance of the NP-bmimTf $\mathrm{N}_{2}$ composite with a gold content of $40 \mathrm{wt} \%$. Although the composite was a black and highly sticky paste in the bulk, it actually appeared to be red when prepared as a thin film wedged between the glass slides (Figure 6b). The red color originates from the plasmon absorption (Figure $6 \mathrm{c}$ ) of individual NPs in the composite, which clearly suggested the NPs were well dispersed in $\operatorname{bmimTf}_{2} \mathrm{~N}$ even at a high concentration. The value of $40 \mathrm{wt} \%$ gold content corresponds to a $5 \mathrm{vol} \%$ volume fraction of gold, in which the average inter-NP distance can be estimated to be $6.3 \mathrm{~nm}$ given a random dispersion. The distance of $6.3 \mathrm{~nm}$ might not be close enough to observe the plasmonic coupling between NPs, ${ }^{49}$ and no apparent peak shift was found in the peak position of the absorption spectrum in comparison with that of a dilute solution in acetone (Figure $6 \mathrm{~b}$ and Figure 1).

The NP-bmimTf $2 \mathrm{~N}$ composite was directly observed by TEM. Because the composite was highly sticky, the sample for TEM was prepared by diluting the composite with a small amount of acetone, followed by casting the acetone solution onto a TEM grid. A certain amount of bmim $\mathrm{Tf}_{2} \mathrm{~N}$ remained on the TEM grid together with the gold NPs because bmimTf ${ }_{2} \mathrm{~N}$ was difficult to evaporate. It should be noted that the composition ratio between the NPs and $\operatorname{bmimTf}_{2} \mathrm{~N}$ was not preserved, because acetone could rinse off a part of the bmimTf $f_{2}$ N. As shown in Figure 7, the gold NPs appeared to aggregate in the droplets of bmimTf $f_{2} \mathrm{~N}$ as the slightly dark background of aggregates. ${ }^{50}$ The aggregate structures of the composite was very different from those obtained for the sample without bmimTf ${ }_{2} \mathrm{~N}$ 
a

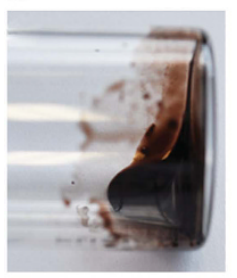

b

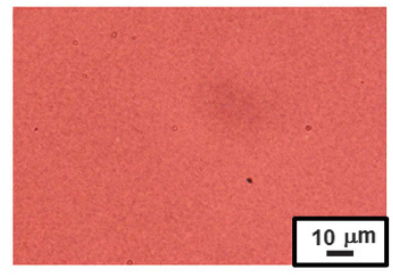

C

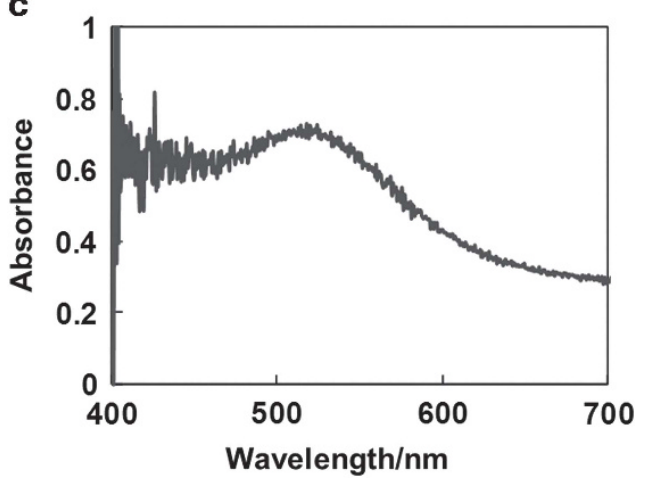

Figure 6 (a) Photograph and (b) optical microscope image of a gold nanoparticle-1-butyl-3-methylimidazolium bis(trifluoromethanesulfonyl)amide composite (gold content $=40 \mathrm{wt} \%$ ) and (c) its absorption spectrum.

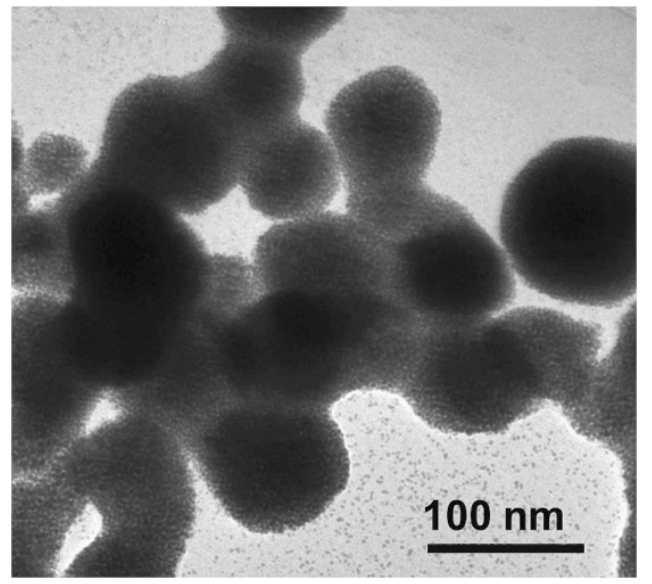

Figure 7 Typical transmission electron microscopy image of a goldnanoparticle-1-butyl-3-methylimidazolium bis(trifluoromethanesulfonyl)amide composite.

(Figure 3). The gold NPs formed no supercrystals but irregularly assembled into lumps. The individual NPs were identified in the aggregates, corresponding to the appearance of the plasmon absorption band for the composite (Figure 6). ILs were reported to form a double layer at a charged surface, ${ }^{51}$ which was also expected to induce the layering of ionic components in an alternate manner. ${ }^{28,52}$ The layering of ionic components was estimated to extend to 10-bilayers. ${ }^{53}$ The cationic gold NPs were therefore expected to be strongly solvated by the formation of a multilayer of ionic components triggered by the ordering of $\mathrm{Tf}_{2} \mathrm{~N}$ anions at the interface, which might also hinder the tight packing of NPs into superlattices via van der Waals interactions between the NPs.

The DMAET-Tf 2 N-capped gold NPs were also well dispersed in a polymerizable IL, MOE-200. The NP-monomer composite was then

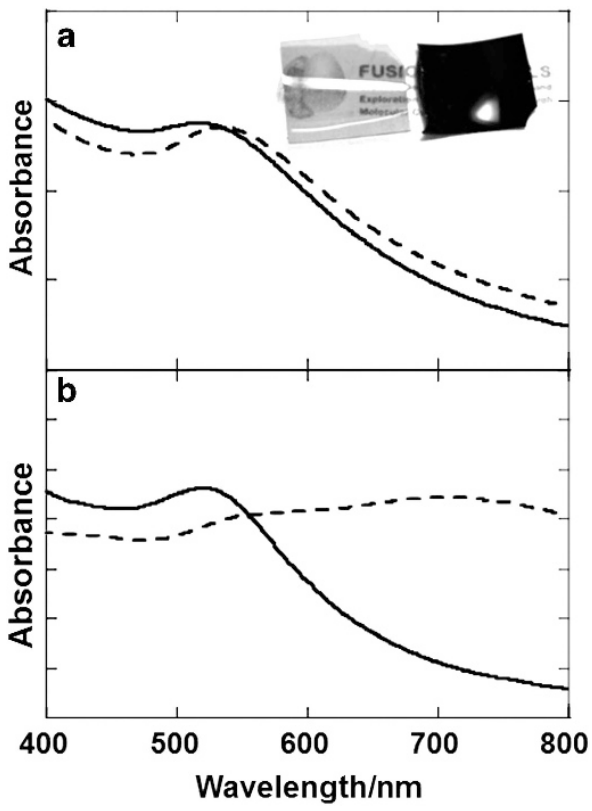

Figure 8 Absorption spectra of gold nanoparticles in polymer (a) and monomer (b) before (solid lines) and after (broken lines) the heat treatment. Inset: picture of the polymer composite films with different gold content.

mixed with a photoinitiator, Irgacure 184, and a cross-linker, diethylene glycol dimethacrylate, followed by irradiating with UV light to give a polymer composite film of NPs with a thickness of $100 \mu \mathrm{m}$. The polymer composite was basically transparent and brown colored with the clear appearance of a plasmon absorption for individual NPs (Figure 8), suggesting the good dispersion of NPs in the IL polymer. The increase in the gold content decreases the transmittance of composite film due to the high optical density of the plasmon absorption. The TG analysis of the polymer composite suggested the film could incorporate the gold to as high as $13 \mathrm{wt} \%$ (Supplementary Figure S2). Although we also tried to prepare the composite film with a gold content of $20 \mathrm{wt} \%$, the high optical density of the film prevented the photopolymerization of the entire film. The gold NPs showed high thermal stability in the polymer. The heat treatment of the monomer solution at $100{ }^{\circ} \mathrm{C}$ for $10 \mathrm{~min}$ resulted in the irreversible agglomeration of NPs (Supplementary Figure S3) and the monomeric composite became opaque with the disappearance of the plasmon absorption band characteristic for gold NPs (Figure 8b). Although the thermal decomposition of the ligand of the NPs started above $300^{\circ} \mathrm{C}$ (Figure 4), the heating might activate the diffusion collision of NPs and the desorption of surface ligands, which induces thermal fusion of gold NPs. By contrast, the heating of the polymeric composite led to only a slight change in the absorption spectrum, even when annealed at $230^{\circ} \mathrm{C}$ for $30 \mathrm{~min}$ (Figure 8a). The suppressed diffusion of NPs immobilized in the solid matrix and the thermal stability of the IL polymer would be responsible for the stability of NPs in the composite.

\section{CONCLUSION}

In conclusion, gold NPs capped with a simplified IL-like ligand were prepared, which were incorporated into the fusion materials with ILs. The high cohesive energy of IL-like ligand drove the assembly of NPs into a glassy amorphous film and superlattices. The DMAET-Tf $2 \mathrm{~N}$ capped gold NPs were well dispersed at concentrations of up to $40 \mathrm{wt}$ $\%$ of inorganic content in $\mathrm{Tf}_{2} \mathrm{~N}$-based ILs. The fusion material of gold 
NPs with an IL monomer was readily converted into a polymeric composite, in which gold NPs showed high thermal stability. We envisage that the IL-like ligand with its simple structure could be a universal ligand for a wide range of inorganic NPs to provide an interface compatible with ILs.

\section{ACKNOWLEDGEMENTS}

This work was supported in part by a Grant-in-Aid for Scientific Research on Innovative Areas of 'Fusion Materials' (Area no. 2206) from the Ministry of Education, Culture, Sports, Science and Technology (MEXT).

1 Parvulescu, V. I. \& Hardacre, C. Catalysis in ionic liquids. Chem. Rev. 107, 2615-2665 (2007).

2 Han, X. \& Armstrong, D. W. Ionic liquids in separations. Acc. Chem. Res. 40, 1079-1086 (2007).

3 MacFarlane, D. R., Pringle, J. M., Howlett, P. C. \& Forsyth, M. Ionic liquids and reactions at the electrochemical interface. Phys. Chem. Chem. Phys. 12, 1659-1669 (2010).

4 Armand, M., Endres, F., MacFarlane, D. R., Ohno, H. \& Scrosati, B. Ionic-liquid materials for the electrochemical challenges of the future. Nat. Mater. 8 621-629 (2009).

5 Smiglak, M., Pringle, J. M., Lu, X., Han, L., Zhang, S., Gao, H., MacFarlane, D. R. \& Rogers, R. D. Ionic liquids for energy, materials, and medicine. Chem. Commun. 50, 9228-9250 (2014).

6 Nakashima, T. \& Kimizuka, N. Interfacial synthesis of hollow $\mathrm{TiO}_{2}$ microspheres in ionic liquids. J. Am. Chem. Soc. 125, 6386-6387 (2003).

7 Antonietti, M., Kuang, D. B., Smarsly, B. \& Yong, Z. Ionic liquids for the convenient synthesis of functional nanoparticles and other inorganic nanostructures. Angew. Chem.-Int. Edit 43, 4988-4992 (2004).

$8 \mathrm{Ma}, \mathrm{Z}$., Yu, J. H. \& Dai, S. Preparation of inorganic materials using ionic liquids. Adv. Mater. 22, 261-285 (2010).

9 Torimoto, T., Tsuda, T., Okazaki, K. \& Kuwabata, S. New frontiers in materials science opened by ionic liquids. Adv. Mater. 22, 1196-1221 (2010).

10 Fukushima, T., Kosaka, A., Ishimura, Y., Yamamoto, T., Takigawa, N., Ishii, N. \& Aida, T. Molecular ordering of organic molten salts triggered by single-walled carbon nanotubes. Science 300, 2072-2074 (2003).

11 Dupont, J. From molten salts to ionic liquids: a "nano" journey. Acc. Chem. Res. 44 1223-1231 (2011).

12 Kato, T. Self-assembly of phase-segregated liquid crystal structures. Science $\mathbf{2 9 5}$, 2414-2418 (2002).

13 Greaves, T. L. \& Drummond, C. J. Ionic liquids as amphiphile self-assembly media. Chem. Soc. Rev. 37, 1709-1726 (2008)

14 Kimizuka, N. \& Nakashima, T. Spontaneous self-assembly of glycolipid bilayer membranes in sugar-philic ionic liquids and formation of ionogels. Langmuir 17, 6759-6761 (2001).

15 Nakashima, T., Zhu, J., Qin, M., Ho, S.-S. \& Kotov, N. A. Polyelectrolyte and carbon nanotube multilayers made from ionic liquid solutions. Nanoscale 2 2084-2090 (2010)

16 Nakashima, T. \& Kimizuka, N. Water/lonic liquid interfaces as fluid scaffolds for the two dimensional self-assembly of charged nanospheres. Langmuir 27, 1281-1285 (2011).

17 Nakashima, T. \& Kimizuka, N. Controlled self-assembly of amphiphiles in ionic liquids and the formation of ionogels by molecular tuning of cohesive energies. Polym. J. 44, 665-671 (2012).

18 Fumino, K., Wulf, A. \& Ludwig, R. Strong, localized, and directional hydrogen bonds fluidize ionic liquids. Angew. Chem. Int. Ed. 47, 8731-8734 (2008).

19 Choudhury, A. R., Winterton, N., Steiner, A., Cooper, A. I. \& Johnson, K. A. In situ crystallization of ionic liquids with melting points below $25^{\circ} \mathrm{C}$. CrystEngComm 8 , 742-745 (2006)

20 Lopes, J. \& Padua, A. A. H. Nanostructural organization in ionic liquids. J. Phys. Chem B 110, 3330-3335 (2006).

21 Triolo, A., Russina, O., Bleif, H. J. \& Di Cola, E. Nanoscale segregation in room temperature ionic liquids. J. Phys. Chem. B 111, 4641-4644 (2007).

22 Scholten, J. D., Leal, B. C. \& Dupont, J. Transition metal nanoparticle catalysis in ionic liquids. ACS Catalysis 2, 184-200 (2012).

23 Dupont, J. \& Scholten, J. D. On the structural and surface properties of transition-meta nanoparticles in ionic liquids. Chem. Soc. Rev. 39, 1780-1804 (2010).

24 Torimoto, T., Okazaki, K., Kiyama, T., Hirahara, K., Tanaka, N. \& Kuwabata, S. Sputter deposition onto ionic liquids: Simple and clean synthesis of highly dispersed ultrafine metal nanoparticles. Appl. Phys. Lett. 89, 243117-1-243117-3 (2006).

25 Le Bideau, J., Viau, L. \& Vioux, A. Ionogels, ionic liquid based hybrid materials. Chem. Soc. Rev. 40, 907-925 (2011).
26 Fukushima, T. \& Aida, T. Ionic liquids for soft functional materials with carbon nanotubes. Chem. Eur. J. 13, 5048-5058 (2007).

27 Bara, J. E., Camper, D. E., Gin, D. L. \& Noble, R. D. Room-temperature ionic liquids and composite materials: Platform technologies for $\mathrm{CO}_{2}$ capture. Acc. Chem. Res. 43, 152-159 (2010).

28 Ueno, K. \& Watanabe, M. From colloidal stability in ionic liquids to advanced soft materials using unique media. Langmuir 27, 9105-9115 (2011).

29 Lu, Y., Das, S. K., Moganty, S. S. \& Archer, L. A. Ionic liquid-nanoparticle hybrid electrolytes and their application in secondary lithium-metal batteries. Adv. Mater. 24 4430-4435 (2012).

30 Bernardi, F., Scholten, J. D., Fecher, G. H., Dupont, J. \& Morais, J. Probing the chemical interaction between iridium nanoparticles and ionic liquid by XPS analysis. Chem. Phys. Lett. 479, 113-116 (2009).

31 Kauling, A., Ebeling, G., Morais, J., Padua, A., Grehl, T., Brongersma, H. H. \& Dupont, J. Surface composition/organization of ionic liquids with au nanoparticles revealed by high-sensitivity low-energy ion scattering. Langmuir 29, 14301-14306 (2013).

32 Itoh, H., Naka, K. \& Chujo, Y. Synthesis of gold nanoparticles modified with ionic liquid based on the imidazolium cation. J. Am. Chem. Soc. 126, 3026-3027 (2004).

33 Tatumi, R. \& Fujihara, H. Remarkably stable gold nanoparticles functionalized with a zwitterionic liquid based on imidazolium sulfonate in a high concentration of aqueous electrolyte and ionic liquid. Chem. Commun. 41, 83-85 (2005).

34 Moganty, S. S., Srivastava, S., Lu, Y., Schaefer, J. L., Rizvi, S. A. \& Archer, L. A. Ionic liquid-tethered nanoparticle suspensions: A novel class of ionogels. Chem. Mater. 24, 1386-1392 (2012).

35 Ueno, K., Sano, Y., Inaba, A., Kondoh, M. \& Watanabe, M. Soft glassy colloidal arrays in an ionic liquid: Colloidal glass transition, ionic transport, and structural color in relation to microstructure. J. Phys. Chem. B 114, 13095-13103 (2010).

36 Bourlinos, A. B., Herrera, R., Chalkias, N., Jiang, D. D., Zhang, Q., Archer, L. A. \& Giannelis, E. P. Surface-functionalized nanoparticles with liquid-like behavior. Adv. Mater. 17, 234-237 (2005)

37 Warren, S. C., Banholzer, M. J., Slaughter, L. S., Giannelis, E. P., DiSalvo, F. J. \& Wiesner, U. B. Generalized route to metal nanoparticles with liquid behavior. J. Am. Chem. Soc. 128, 12074-12075 (2006).

$38 \mathrm{Kim}$, Y., Kim, D., Kwon, I., Jung, H. W. \& Cho, J. Solvent-free nanoparticle fluids with highly collective functionalities for layer-by-layer assembly. J. Mater. Chem. 22 11488-11493 (2012).

39 Nakashima, T. \& Kawai, T. Quantum dots-ionic liquid hybrids: Efficient extraction of cationic CdTe nanocrystals into an ionic liquid. Chem. Commun. 1643-1645 (2005).

40 Nakashima, T., Sakakibara, T. \& Kawai, T. Highly luminescent CdTe nanocrystalpolymer composites based on ionic liquid. Chem. Lett. 34, 1410-1411 (2005).

41 Nakashima, T., Nonoguchi, Y. \& Kawai, T. Ionic liquid-based luminescent composite materials. Polym. Adv. Technol 19, 1401-1405 (2008).

42 Liu, X. M., Tomita, Y., Oshima, J., Chikama, K., Matsubara, K., Nakashima, T. \& Kawai, T. Holographic assembly of semiconductor cdse quantum dots in polymer for volume Bragg grating structures with diffraction efficiency near $100 \%$. Appl. Phys. Lett. 95, 261109 (2009).

43 Liu, X. M., Adachi, Y., Tomita, Y., Oshima, J., Nakashima, T. \& Kawai, T. High-order nonlinear optical response of a polymer nanocomposite film incorporating semiconducotor CdSe quantum dots. Opt. Express 20, 13457-13469 (2012).

44 Ono, T., Ohta, M. \& Sada, K. Ionic polymers act as polyelectrolytes in nonpolar media. ACS Macro Lett 1, 1270-1273 (2012).

45 Templeton, A. C., Pietron, J. J., Murray, R. W. \& Mulvaney, P. Solvent refractive index and core charge influences on the surface plasmon absorbance of alkanethiolate monolayer-protected gold clusters. J. Phys. Chem. B 104, 564-570 (2000)

46 Israelachvili, J. Intermolecular and Surface Forces, 2nd edn (Academic Press: San Diego, CA, USA, 1991).

47 Yonezawa, T., Onoue, S.-i. \& Kimizuka, N. Metal coating of DNA molecules by cationic metastable gold nanoparticles. Chem. Lett. 31, 1172-1173 (2002).

48 Fredlake, C. P., Crosthwaite, J. M., Hert, D. G., Aki, S. N. V. K., Brennecke, J. F. Thermophysical properties of imidazolium-based ionic liquids. J. Chem. Eng. Data 49, 954-964 (2004).

49 Zhong, Z., Patskovskyy, S., Bouvrette, P., Luong, J. H. T. \& Gedanken, A. The surface chemistry of Au colloids and their interactions with functional amino acids. J. Phys. Chem. B 108, 4046-4052 (2004).

50 Ueno, K., Inaba, A., Kondoh, M. \& Watanabe, M. Colloidal stability of bare and polymer-grafted silica nanoparticles in ionic liquids. Langmuir 24, 5253-5259 (2008).

51 Sha, M., Wu, G., Dou, Q., Tang, Z. \& Fang, H. Double-layer formation of [Bmim] $\left[\mathrm{Pf}_{6}\right]$ ionic liquid triggered by surface negative charge. Langmuir 26, 12667-12672 (2010).

52 Hayes, R., Warr, G. G. \& Atkin, R. At the interface: Solvation and designing ionic liquids. Phys. Chem. Chem. Phys. 12, 1709-1723 (2010).

53 Ueno, K., Kasuya, M., Watanabe, M., Mizukami, M. \& Kurihara, K. Resonance shear measurement of nanoconfined ionic liquids. Phys. Chem. Chem. Phys. 12 4066-4071 (2010).

Supplementary Information accompanies the paper on Polymer Journal website (http://www.nature.com/pj) 